\title{
UTILIZACIÓN DEL TRANSPORTE INTERMODAL PARA MEJORAR LA COMPETITIVIDAD Y EL DESARROLLO: CORREDOR BOGOTÁ-BELENCITO
}

\author{
USE OF INTERMODAL TRANSPORT TO IMPROVE COMPETITIVENESS AND \\ DEVELOPMENT: BOGOTÁ-BELENCITO CORRIDOR
}

Luz Rocío Corredor González*

Ana María Alejandra Prieto Marín**

Linda Bibiana Fernández Guerrero****

Recibido: 17 de julio de 2017

Aceptado: 25 de octubre de 2017

\section{Resumen}

El departamento de Boyacá es conocido por la diversidad de su territorio gracias a su posición geográfica, la cual le ha permitido tener una vocación agrícola, en la que predomina la producción de tipo familiar. La región en esta materia, cuenta con ventajas comparativas y competitivas, que al mismo tiempo se encuentran limitadas por diversas problemáticas, razón por la que se realizó un inventario de las potencialidades agrícolas con el fin de establecer propuestas de política y de transporte que lleve a que el sector agrícola eleve su productividad y competitividad, sin desconocer la heterogeneidad y la diversidad que caracteriza a este departamento.

Palabras clave: agricultura, asociatividad, competitividad, integración regional, transporte intermodal.

\begin{abstract}
The department of Boyacá is known for the diversity of its territory thanks to its geographical position, which has allowed it to have an agricultural vocation, in which family-type production predominates. The region has comparative and competitive advantages, which at the same time, are limited by various problems, which is why an inventory of agricultural potentialities was made in order to establish policy and transport proposals, that lead the farming sector to increase its productivity and competitiveness, without ignoring the heterogeneity and diversity that characterizes this department.
\end{abstract}

Keywords: agriculture, associativity, competitiveness, regional integration, intermodal transport.

\footnotetext{
* Economista, doctorante en Estudios Políticos. Docente investigadora, Facultad de Economía, Fundación Universidad de América, Bogotá, Colombia. ORCID: http://orcid.org/0000-0001-7453-2622.luz.corredor@profesores. uamerica.edu.co

** Economista, Fundación Universidad de América, Bogotá, Colombia. anamariaalejandra15@gmail.com

*** Economista, Fundación Universidad de América, Bogotá, Colombia. bibifernandez38@ gmail.com
} 


\section{INTRODUCCIÓN}

El departamento de Boyacá es conocido por la diversidad de su territorio y por su tradición agrícola. No obstante, esta región enfrenta problemas de baja productividad y competitividad principalmente a nivel de los pequeños productores, debido a que no hay suficientes centros de acopio que se adapten a las necesidades requeridas y a los estándares internacionales. Asimismo, muchas de las asociaciones cooperativas de estos se encuentran dispersas y desarticuladas, lo cual le impide responder de manera adecuada a los retos de la competitividad. Adicionalmente, existe una débil infraestructura vial terciaria que comunica a los municipios con las principales ciudades del departamento, que sumada a los precios de combustibles elevados, provoca que los costos logísticos asociados al transporte sean mayores, afectando significativamente la competitividad del sector.

Por ello se propone la ejecución de una hoja de ruta que contribuya a mejorar la situación existente, impulsando la optimización de mecanismos para el acceso básico de capital para la agricultura, identificando nuevas alternativas y herramientas que fortalezcan la asociatividad de los pequeños productores agrícolas (grupos de productores por producto en común), como el sistema intermodal de transporte (articulando el sistema férreo con el carretero). Implementar las estrategias propuestas facilitará a los pequeños agricultores aumentar su competitividad, su productividad y reducir los costos de transporte.

De esta forma, se inicia con la realización de un inventario de las potencialidades productivas del departamento, identificando los municipios que tienen una producción superior a las 1000 t/año en productos tales como la caña panelera, el tomate, la cebolla de bulbo y de rama, el plátano, la yuca, la pera y el maíz, y señalando las principales problemáticas que le impiden a la región aprovechar estas potencialidades.

A partir de esto, se analizan los objetivos de integración regional propuestos en los planes de desarrollo y en los planes indicativos de estos municipios seleccionados y del departamento, con el fin de contribuir A la construcción de la hoja de ruta a seguir. Así, en la tercera parte se generan las propuestas de la política que permitirá al sector superar sus debilidades y aprovechar las potencialidades para ser más competitivo.

\section{Metodología}

Este artículo es el resultado de una investigación descriptiva, cualitativa y cuantitativa. Inicialmente se realizó un inventario de las potencialidades productivas del departamento de Boyacá, con base en la caracterización social, territorial y de producción de los departamentos y municipios de Colombia realizada por el Dirección de Desarrollo Rural Sostenible y el Departamento Nacional de Planeación (DDRS Y DNP) entre los años 2013 y 2014, del cual se eligieron ocho productos transables en el mercado internacional; luego se identificaron los veinte municipios con una producción mayor a 1000 toneladas anuales de los ocho productos que se escogieron. No se tuvieron en cuenta los municipios de Cundinamarca que hacen parte del corredor férreo, ya que uno de los objetivos de la investigación es que Boyacá sea la despensa agroalimentaria de Bogotá.

Terminada esta etapa, se evidencia que el departamento sí tiene potencial agrícola, por lo que podría implementarse la estrategia de que los productos sean transportados en dos vagones del tren. Luego, a partir de los Planes Indicativos reportados al (DNP) 2016-2017 de estos 20 municipios, se construye una base de datos para reconocer las metas que involucren temas de integración regional, asociatividad, ayuda a pequeños productores, mercadeo, comercialización, medio ambiente e infraestructura.

Una vez realizado el inventario agrícola junto con el análisis de los objetivos de integración regional propuestos en los planes de desarrollo locales y regionales, se procedió a estructurar la hoja 
de ruta según el círculo virtuoso de cuatro dimensiones: a) política agrícola, b) infraestructura c) estructura de mercado y d) herramientas innovadoras; realizado por el Foro Económico Mundial y McKinsey Company en el año 2010.

Para desarrollar cada dimensión se tuvieron en cuenta las herramientas de análisis ya existentes, y se incluyeron nuevas propuestas para contribuir al impulso de la competitividad de los pequeños productores en los veinte municipios objeto de estudio, tales como políticas, asociatividad, apoyo a los productores, así como la implementación de un sistema intermodal que involucra el transporte férreo y el carretero (camiones y camionetas), el cual minimiza los costos de transporte aportando al aumento de la competitividad.

\section{Resultados}

\section{Potencialidades agrícolas}

En la investigación se encontró que efectivamente el departamento de Boyacá cuenta con un potencial agrícola y que los principales municipios que cumplen con una alta producción según los ochos productos que se escogieron, son: Aquitania, Campohermoso, Duitama, Guateque, Jenesano, Maripí, Moniquirá, Nobsa, Nuevo Colón, Paipa, Pauna, Ráquira, Sáchica, Sogamoso, Sutamerchán, Tibaná, Tibasosa, Tota, Tunja y Villa de Leyva. En estos se evidencia una producción anual igual o superior a las 1000 toneladas en caña panelera, tomate, cebolla de bulbo, cebolla de rama, plátano, yuca, pera, maíz y caña miel; tal información fue tomada de la caracterización social, territorial y de producción de los departamentos y municipios de Colombia realizada por (DDRS) y (DNP) y publicada en el Anuario estadístico del sector agropecuario 2013.

Según esta información, tenemos como resultado que la cebolla de bulbo es el principal producto cultivado en Tibasosa; en Pauna es el cultivo de caña panelera; en cuanto al tomate, Villa de Leyva concentra la mayor parte de la producción seguida por Sáchica; la cebolla de rama se produce en Aquitania y representa ocho veces la producción del municipio de Tota; el plátano se produce en Pauna, Moniquirá y Campohermoso; la pera en Tibaná, Jenesano y Nuevo Colón; la yuca en Campohermoso, Maripí y Moniquirá, y por último, la producción de maíz se concentra en los municipios de Moniquirá y Ráquira.

En cuanto a la composición del PIB del departamento de Boyacá, el sector agrícola (incluyendo la ganadería, la caza y la pesca) participó en promedio con un $13.6 \%$ durante el periodo 2010-2014, situación que refleja la necesidad de generar valor agregado en este sector para que este porcentaje aumente, desde alternativas que fomenten nuevas oportunidades productivas con el fin de ampliar las capacidades de los habitantes y así fortalecer las ventajas competitivas.

En el plan de Desarrollo Departamental vigente, se evidencia que la productividad del sector está afectada por la baja tecnificación, con valores por debajo del promedio nacional. Por ejemplo, el porcentaje de uso de maquinaria en el sector agropecuario en Boyacá es del $5.1 \%$ frente al $16.6 \%$ del promedio nacional; el de construcciones agropecuarias es del $5 \%$ frente al $16.8 \%$ del promedio nacional; y en cuanto a sistemas de riego es $1.8 \%$ del área cultivada, que también está muy por debajo del promedio nacional. La falta de asistencia técnica, incentivos, y planificación del territorio, y la excesiva intermediación en los canales de comercialización, son factores que afectan la productividad, además la mayoría de los predios se encuentran en falsa tradición y un significativo porcentaje de los productores son mini y microfundistas, por lo cual, se dificulta el acceso a créditos y certificaciones que les permitan encontrar financiación (Gobernación de Boyacá, 2016).

Otra problemática es la que plantea, Carolina Cárdenas (2015), quien en su investigación de trabajo de grado en Relaciones Internacionales, Seguridad y Estrategia, titulada Exportación de productos no tradicionales del departamento de Boyacá, referencia que el departamento carece de vías 
terciarias en buen estado, lo cual dificulta la comunicación entre los municipios y su capital, que a su vez se refleja en el incremento de los costos de logística, porque las empresas de carga aumentan sus tarifas debido al estado de las vías que representa un desgaste para sus vehículos. Adicionalmente, el departamento carece de centros de acopio, lo cual dificulta la operación logística y la hace más costosa, restándole competitividad al producto y eficiencia a la operación (Cárdenas, 2015). Esto genera el incremento del número de intermediarios a lo largo de la cadena de suministro para hacer llegar los productos a la capital, disminuyendo los niveles de rentabilidad para los pequeños productores. Por ello, si se pretende aprovechar las potencialidades, se deben resolver los problemas mencionados adicionando el fomento de la asociatividad entre los pequeños productores.

\section{Integración regional}

Para la investigación se hizo énfasis en la integración regional económica de este sector, en la medida en que el mercado de productos agrícolas de Boyacá tiene el potencial para trascender las fronteras municipales y departamentales si se diseñan e implementan mecanismos que permitan aprovechar las economías de escala que surgen con el comercio, ganar aprendizajes en productividad, potenciar la asociatividad, y optimizar las ventajas de un sistema multimodal de transporte que beneficie a los pequeños productores. La integración regional puede ayudar a los municipios a incorporar formas más eficientes de producción y especialización, con un mayor componente tecnológico en los procesos de producción y distribución que redundaría en valor agregado.

Desde luego, la integración regional agrícola parte de que los municipios y el departamento generen una correcta sinergia a través de la implementación de políticas agricolas comunes, basadas en elementos coherentes como son una visión compartida de futuro, objetivos comunes de oferta agrícola, instrumentos y medidas de implementación de las políticas y mecanismos de financiación a la producción y distribución de los productos agrícolas.

En Boyacá, la agricultura no puede estar separada de los asuntos políticos y de la comercialización, dado que los intereses compartidos son cruciales para lograr acuerdos entre los productores de un mismo municipio como también entre aquellos de diferentes municipios. De hecho, la asociatividad intra e intermunicipal puede reducir conflictos por cuotas de comercio entre vecinos y contribuir a mitigar riesgos ocasionados por choques externos, lo que, sumado a buenas estrategias de negociación, puede generar coaliciones potentes para aumentar la producción agrícola y llevar los productos a los departamentos vecinos.

Para ello se busca orientar al pequeño productor a no solamente satisfacer el mercado interno, sino expandir su actividad económica fuera de la frontera de su territorio, en un marco de mayor competitividad, con un sistema de acopio y transporte multimodal para sus productos, una estrategia de reducción de costos de transacción (como impuestos adicionales o tarifas al productor por el comercio intermunicipal e interdepartamental) y un acceso a la mayor cantidad de información posible (para reducir las asimetrías de información), lo cual puede generar un efecto positivo en su propio ingreso, y de esta forma mejorar los niveles de desarrollo local y de expansión de la frontera agrícola.

Para identificar los objetivos de integración regional se extrajo información de las metas propuestas por los planes de desarrollo municipales y departamental, así como de los planes indicativos en los temas relacionados con la integración regional, la asociatividad, el mercadeo y la comercialización, la infraestructura vial y de transporte, el ordenamiento territorial, la capacitación y asistencia técnica, el apoyo a los productores y el medio ambiente. En este análisis se evidenció que las metas de cada municipio suelen estar aisladas y no se relacionan con las necesidades generales del departamento; en cuanto a metas referidas a la asociatividad, se encontraron 33 metas de producto de los municipios y 1 del departamento. Estas incluyen crear nuevas asociaciones y fortalecer las existentes, así como realizar diagnósticos de organizaciones vigentes, crear asociaciones de 
familias productoras, patrocinar asociaciones, financiar proyectos de alianzas productivas y vincular productores a asociaciones. El problema de estas estrategias radica en que son planeadas exclusivamente para cada uno de los municipios y no buscan establecer una conexión con otros municipios que producen lo mismo, por ende, no se logra obtener una correcta sinergia que permita el aprovechamiento máximo de cada producto y así el mayor desarrollo económico.

En la conformación de mercados es clave la organización y realización de ferias y ruedas de negocios por parte de entes gubernamentales como las Secretarías de Agricultura y Fomento Agropecuario, así como de las Cámaras de Comercio, para facilitar la comercialización de productos agrícolas y la creación de esquemas de agricultura sostenible que incluyan la planeación agrícola asociativa, la construcción de centros de acopio, la construcción y mantenimiento de infraestructura y la comercialización. 49 estrategias de este tipo son municipales y una departamental, y su desarrollo se puede ver limitado por la poca asociatividad entre municipios para los diferentes productos, razón por la cual es de vital importancia impulsar y favorecer proyectos de asociatividad intermunicipal.

En la construcción y mantenimiento de infraestructura vial y de transporte, se destacan 148 metas estratégicas compuestas por 8 del departamento y las restantes 140 de los municipios. Se incluye ampliación del transporte público en los municipios, manejo de aguas lluvias en corregimientos, señalización en las vías de comunicación, planes viales regionales, seguridad vial, planes de transporte, adquisición de maquinaria para el mantenimiento de las vías, inventarios de vías, planes de movilidad, estudios de transporte municipal, adecuación de terminales de transporte, construcción de placa huella, vías rurales y transporte para la comercialización, entre otros. Aunque se plantea el fortalecimiento de la infraestructura vial secundaria y terciaria, no se evidencian estrategias para la implementación de un sistema de transporte multimodal, razón por la cual se ven afectadas las posibilidades de integración entre municipios, lo que a su vez genera una pérdida en los niveles de competitividad del sector agrícola.

En el ordenamiento territorial se encontraron 23 propuestas de los municipios y 9 del departamento. Guateque, Nobsa, Paipa, Tibaná y Tota no presentan metas relacionadas con el ordenamiento territorial, el cual es importante para atraer inversión privada, mejorar usos del suelo y elevar los niveles de competitividad. El departamento hace un uso adecuado del $50 \%$ del suelo, por ende, las posibilidades de elevar la competitividad y la integración regional van a estar condicionadas por la claridad sobre el ordenamiento territorial y sus reglamentaciones adecuadas en cabeza de los alcaldes y del gobernador del departamento.

En la capacitación y asistencia técnica se presentan 47 metas municipales y 16 departamentales, encaminadas a brindar asistencia técnica especializada y capacitaciones con el fin de modernizar los procesos agropecuarios, conformar alianzas interinstitucionales, formar competencias, establecer un sistema de información agropecuaria y crear fondos para la asistencia técnica a productores. Estas metas deben estar acompañadas de procesos asociativos entre los municipios productores para ser más eficientes y tener mayor impacto.

En apoyo a los productores se presentan 71 metas municipales y 30 departamentales, las cuales buscan promover y acompañar proyectos productivos, orientar al productor sobre el uso y manejo del recurso hídrico, proveer subsidios y acceso a créditos, generar valor agregado en la producción, mejorar la competitividad, crear bancos de semillas, establecer programas de apoyo para los agricultores, implementar estrategias de desarrollo rural, promover viveros, crear marcas o sellos de origen, promover la producción para la seguridad alimentaria, definir cadenas de valor en la producción agrícola, suministrar maquinaria, construir distritos de riego, recopilar buenas prácticas agrícolas, realizar estudios de producción, financiar a los pequeños productores y promover la crea- 
ción de microempresas. Para tener una ejecución más eficiente de estos planes, se hace necesaria la implementación de una estrategia que incentive la asociatividad inter e intramunicipal.

Las estrategias relacionadas con el medio ambiente son las de mayor representatividad. Se presentan 160 metas municipales y 47 departamentales encaminadas a la educación ambiental, la preservación y recuperación de fuentes hídricas, el manejo de residuos, la prevención de desastres, la creación de sellos verdes, el transporte no contaminante, la mitigación del cambio climático y la elaboración de agendas ambientales.

Sólo el municipio de Duitama cuenta con una estrategia encaminada a la integración regional. La ausencia de este tipo de estrategias exige el compromiso de la institucionalidad púbica departamental para promover alianzas entre municipios según afinidad de productos agrícolas; asimismo, reforzar con mayor eficiencia los mecanismos de asociatividad promovidos tanto en las esferas locales como por la intermediación del departamento, de manera que los municipios con afinidad productiva puedan encontrar mayores espacios de integración regional para aunar esfuerzos orientados a aumentar la productividad, expandir las fronteras de comercio y elevar los ingresos, satisfaciendo no solo la demanda interna sino también la de otras entidades territoriales, principalmente la de Bogotá. Aunque se evidencian estrategias de infraestructura vial y de transporte, estas se relacionan con vías terrestres, placas huella, señalización y comunicación veredal, persistiendo la ausencia de un enfoque de transporte multimodal, lo que limita las posibilidades de competitividad agrícola de los municipios objeto del estudio.

Teniendo en cuenta lo anterior, se recomienda actualizar los Planes de Ordenamiento Territorial y los Esquemas de Ordenamiento Territorial, ya que de lo contrario se reducen las posibilidades de integración y de incrementar la competitividad del sector. Si se actualizan, se podrá además atraer inversión privada y mejorar el uso de los suelos, contribuyendo a mejorar la competitividad y el crecimiento económico de la región. También se recomienda que los gobiernos municipales y el gobierno departamental dispongan de personal que sea proactivo y lo suficientemente capacitado para gestionar recursos y proyectos, con el propósito de brindarle a los productores un acompañamiento de tiempo completo (antes, durante y después de la ejecución de los proyectos) de tiempo completo, y de esta forma mantener los resultados obtenidos a través del tiempo, implementando un desarrollo sostenible con impactos de mediano y largo plazo.

\section{Hoja de ruta}

Se evidenció que el departamento sí cuenta con un potencial agrícola suficiente para consolidarse como despensa alimentaria de la ciudad de Bogotá, D. C. Sin embargo, para que esto pueda concretarse y proyectarse como una realidad, se requiere dar una orientación a los pequeños productores sobre alternativas que les permitan elevar sus niveles de productividad y competitividad, y así generar excedentes para el mercado bogotano.

Esta hoja de ruta se enmarca en un círculo virtuoso de cuatro dimensiones que, desarrolladas adecuadamente y de forma integral, redundarán en beneficios importantes para la producción agropecuaria y generarán mejores oportunidades para los pequeños productores (World Economic Forum, McKinsey y Company, 2010).

La primera dimensión es la de política agrícola; la segunda es la de infraestructura; la tercera se relaciona con la estructura de mercado; y la cuarta es la referente a herramientas innovadoras. Estas dimensiones se interrelacionan, por lo que cada una de ellas depende de las otras y viceversa, es decir, si se define una adecuada política agrícola, ello conducirá a generar la infraestructura necesaria y correcta para elevar la competitividad y la productividad, lo que facilitará hacer los arreglos necesarios para crear una estructura de mercado acorde con las herramientas diseñadas y aplicadas en función de los pequeños productores. 
En cuanto a la política con visión 2032, se propone al departamento de Boyacá tener en cuenta los ejes de economía campesina, costos de producción, desarrollo rural, sostenibilidad ambiental, seguridad alimentaria e integración horizontal y vertical como base para la formulación de planes, programas y proyectos que estarán encaminados a elevar la productividad del sector agrícola. Se debe impulsar el desarrollo de infraestructura a través de la provisión de bienes públicos especiales para el sector agrícola (distritos de riego y drenaje, vías, sistema de transporte, centros de acopio, acceso a servicios públicos y TIC).

Por ello, es necesaria la implementación de un sistema de transporte intermodal (carreteroférreo), el cual les permitirá reducir costos a todos los productores y obtener mayor rentabilidad a los pequeños. Es decir, en la dimensión de infraestructura se resalta la importancia de dar uso al sistema férreo disponible, que es el corredor de Bogotá-Belencito relacionándolo con el carretero por medio dela implementación de un sistema de transporte intermodal. De esta manera los productores de los municipios objeto de estudio deben llegar por carretera a los dos centros de acopio propuestos ubicados en Tunja y Sogamoso, donde los productos serán puestos en los vagones del actual tren que hoy en día transporta carbón, cemento y acero. Si se lleva a cabo esta propuesta se podrán transportar mayores volúmenes de productos por viaje, el medio ambiente se verá beneficiado por el uso del tren debido a una menor contaminación, y podrán generarse complementariedades entre sistemas de camiones y cargue del tren. Un análisis de los costos de transporte ferroviario para el tramo Sogamoso-Bogotá revela que existen importantes ahorros en los costos para el abastecimiento alimentario de Bogotá, lo que se traduce en potenciales beneficios a los pequeños productores, al mejorar la eficiencia y estimular la mayor producción.

Para la estimación de la tarifa de tren, la investigación se apoyó en la Resolución 184 del 16 de enero de 2009, la cual reglamenta el régimen de tarifas que corresponden a los operadores y usuarios de transporte ferroviario de carga y pasajeros en el corredor central. La Resolución basa su dictamen en estudios realizados por el Instituto de Concesiones (INCO). El artículo 2 de la Resolución contiene las tarifas máximas que podrá cobrar el concesionario por el uso de la red férrea en el corredor por tonelada en el año 2009, mientras que el artículo 4 plantea que las tarifas se ajustarán al inicio de cada año con base a la variación del Índice de Precios al Consumidor (IPC), certificada por el DANE para el año inmediatamente anterior. Así, para el año 2017 las tarifas se incrementarán con base en el IPC del 2016. Aplicando esta fórmula se obtienen las siguientes tarifas:

Se toma la suma de $\$ 22.44 \mathrm{t} / \mathrm{km}$ en el año 2009, que para el 2017 ajustada por IPC sería de $\$ 29,94 \mathrm{t} / \mathrm{km}$, siempre que en el corredor Sistema Ferroviario Central se estén transportando hasta un máximo de 2000000 de toneladas anuales. De tal forma, si se tiene que el tramo de Bogotá a Belencito consta de $317 \mathrm{~km}$, el costo total aproximado del transporte férreo por tonelada es de $\$ 9491$ (lo que cuesta llevar una tonelada de Belencito a Bogotá), y si se destinan por día tres vagones, que equivalen a cerca de 100 toneladas, el valor de un viaje en tren sería aproximadamente de \$949063. En comparación, esta misma carga por transporte carretero cuesta alrededor de $\$ 3.248 .190$ (correspondiente a tres tracto camiones que llevan 34 toneladas cada uno, con un costo aproximado de \$1 082730 por el viaje de Sogamoso a Bogotá), por lo tanto si se llevan los productos en tren, los pequeños productores se ahorrarían un $70 \%$ del costo que actualmente les representa llevar sus productos del centro de acopio propuesto a la ciudad de Bogotá.

En la estructura de mercado es necesaria la creación de ocho asociaciones (las asociaciones y los funcionarios públicos deben tener una gran capacidad de liderazgo y gestión). Una vez construidos los dos centros de acopio ubicados en Tunja y Sogamoso para dar aprovechamiento a las potencialidades agrícolas de los municipios objeto de estudio, si las alcaldías implementan óptimas estrategias de divulgación de la importancia de la asociatividad para los pequeños productores, se podrán constituir estas asociaciones, donde los pequeños productores conocerán los 
beneficios de pertenecer a ellas y las formas de financiación a las cuales podrán acceder para mejorar su proceso de producción. En cuanto al desarrollo de herramientas innovadoras, es necesario que las administraciones municipales y la gobernación lleven a cabo un fortalecimiento al interior de sus instituciones para lograr arreglos institucionales entre los diferentes niveles de gobierno.

Si esta hoja de ruta se implementa de manera estratégica, el departamento no solo elevará los niveles de competitividad, sino que podrá convertirse en la despensa agrícola de la ciudad de Bogotá. Así, aumentarán los ingresos de los pequeños productores, lo cual les facilitará el acceso a maquinaria y equipo para dar un valor agregado mayor a la producción agrícola de los municipios objeto de estudio, que aumentarán su productividad y podrán llegar a comercializar progresivamente a diferentes municipios del país.

Para lograr ésta meta, los municipios objeto de estudio deben tener en cuenta los siguientes aspectos:

- El fortalecimiento de las capacidades institucionales privadas y públicas

- El establecimiento de alianzas con socios estratégicos

- La evaluación permanente de la implementación de la hoja de ruta

- El desarrollo de forma coordinada de los distintos roles en la hoja de ruta distribuidos entre responsables, recursos e indicadores de medición del éxito de las estrategias.

- El liderazgo fuerte para mantener el impulso, consolidación y proyección de las estrategias.

En esta hoja de ruta se enfatiza en recomendaciones como la implementación de un sistema de transporte intermodal (carretero, férreo) que permitirá a los pequeños productores reducir sus costos e incrementar el margen de rentabilidad, a la vez que contribuirá a mitigar el impacto ambiental.

Para ello es fundamental que se creen las asociaciones y que estas se coordinen perfectamente con los gobiernos locales y con el gobierno departamental, para incrementar significativamente la competitividad, el crecimiento y desarrollo económico sostenible del departamento. Se recomienda fortalecer institucionalmente a las alcaldías y a la gobernación, ya que esta institucionalidad contribuye a mejorar la eficiencia de la producción, distribución y consumo de los productos agrícolas, en conjunto con políticas dirigidas a mejorar la calidad de vida de los productores. Razón por la cual, el gobierno debe generar incentivos a los productores para que faciliten la transformación productiva y eleven la competitividad. Y se recomienda desde un principio la orientación al pequeño productor para no solo producir para satisfacer el mercado interno, sino también para propiciar su actividad económica fuera de la frontera de su propio territorio, en un marco de mayor competitividad, con un sistema de acopio y transporte multimodal de sus productos, una estrategia de reducción de costos de transacción (como impuestos adicionales o tarifas al productor por el comercio intermunicipal e interdepartamental) y la producción de la mayor cantidad de información posible al pequeño productor (para reducir las asimetrías de información), todo esto redundará en un efecto positivo para su propio ingreso, logrando así mejorar los niveles de desarrollo local y de expansión de la frontera agrícola.

\section{Conclusiones}

A partir de la caracterización social, territorial y de producción de los departamentos y municipios de Colombia realizada por la Dirección de Desarrollo Rural Sostenible (DDRS), y el Departamento Nacional de Planeación (DNP), en el Anuario estadístico del Sector Agropecuario 2013, se evidencia que Boyacá si tiene un potencial agrícola, por lo que se visualiza a este departamento como despensa agroalimentaria de la ciudad de Bogotá. 
Para que se puedan aprovechar estas potencialidades, es necesario la solución de algunas problemáticas que limitan el proceso productivo, tales como la deficiencia de la capacitación y la asistencia técnica a los pequeños productores, así como en el uso inadecuado del suelo y las dificultades en el acceso y la implementación de nuevas y adecuadas tecnologías para los procesos productivos.

La integración regional a favor de la producción agrícola se generará a partir de la creación de asociaciones de pequeños productores y el mercadeo y la comercialización asociados con la construcción de centros de acopio y plazas de mercado, así como con la organización y participación en ferias y ruedas de negocio.

Asimismo, es determinante el mejoramiento de la infraestructura vial y de transporte de los productos agrícolas, así como la reestructuración de los Planes de Ordenamiento Territorial y Esquemas de Ordenamiento Territorial para que exista información actualizada sobre el uso del suelo del departamento.

Desde la creación de asociaciones según el tipo y perfil de producto, la hoja de ruta dará alternativas que buscan elevar la competitividad a partir de la formulación e implementación de una política agrícola común, una mejor infraestructura para que los municipios puedan transportar sus productos de manera eficiente y eficaz a los dos centros de acopio propuestos (en Tunja y Sogamoso) y así poder transportarlos en tren hasta la ciudad de Bogotá, una estructura de mercado donde se evidencien las diferentes opciones de financiación y desde herramientas innovadoras donde las asociaciones puedan ofertar directamente sus productos, eliminando así por lo menos en gran medida la intermediación.

El transporte de las 100 toneladas diarias tiene un costo total de $\$ 949063$ por tren versus $\$ 3.248 .190$ por medio del transporte carretero, el cual necesitaría de tres tracto camiones que lleven $34 \mathrm{t} /$ cada uno, por lo que se obtendría un ahorro del 70\% del transporte de los productos de los centros de acopio a la ciudad de Bogotá. En este cálculo no se tienen en cuenta los costos de transporte carretero que se utilizará de los municipios a los dos centros de acopio.

Las políticas públicas de los entes territoriales, así como las iniciativas privadas, junto al liderazgo y compromiso de los distintos actores de la cadena de producción agrícola, y el conocimiento y aprovechamiento de las herramientas e incentivos existentes ofrecidos por el gobierno central y la cooperación internacional, harán realidad el sueño de una Boyacá verde, especializada en productos orgánicos de fácil comercialización y posicionamiento, no sólo a nivel local sino regional, nacional e internacional, que pueda convertirse en ejemplo para otros departamentos provocando una dinámica con efecto dominó sobre la economía nacional para hacerla próspera y articularla a la política comercial del país.

Si se realiza la implementación del sistema férreo ya existente para el transporte de productos agrícolas hacia la ciudad de Bogotá, los pequeños productores podrán obtener una ventaja, ya que recibirán más dinero por sus productos, y de esta manera podrán aumentar su competitividad y así destinar parte de sus ganancias a mejorar sus procesos productivos.

\section{RefERENCIAS}

Albarracín, L. (2015). Propuesta para el mejoramiento del proceso de comercialización de la cebolla larga en el municipio de Aquitania como estrategia para incrementar su nivel de ventas (tesis). Especialización en Gerencia Estratégica de Mercadeo, Universidad Nacional Abierta y a Distancia, Boyacá, Colombia.

Alcaldía de Campohermoso. (2016). Plan de desarrollo de Campohermoso 2016-2019. Recuperado de http://www.campohermoso-boyaca.gov.co 
Alcaldía de Guateque. (2016). Ciudad de Luz. Plan de desarrollo Guateque Boyacá 2016-2019. Recuperado de http://www.guateque-boyaca.gov.co/Transparencia/PlaneacionGestionyControl/Plan\%20Desarrollo.pdf

Alcaldía de Maripí. (2016). Plan de desarrollo Maripi 2016 - 2019. Recuperado de http://www. maripi-boyaca.gov.co/index.shtml

Alcaldía de Moniquirá. (2016). Plan de desarrollo municipal de Moniquirá 2016-2019 (Acuerdo 011 del 2016). Recuperado de www.moniquirá-boyaca.gov.co/Programación-Plan-de-Desarrollo-Municipal.aspx.

Alcaldía de Pauna. (2016). Plan municipal de desarrollo de Pauna 2016-2019. Recuperado de www. pauna-boyaca.gov.co.

Alcaldía de Sogamoso.(2016). Plan desarrollo 2016-2019. Sogamoso incluyente 2016-2019. Recuperado de http://sogamoso-boyaca.gov.co/apc-aa-files/65616664353566303162646663663062/ plan-desarrollo-municipal-sogamoso-incluyente-2016.pdf

Alcaldía de Sutamarchán. (2016). Plan de desarrollo Sutamarchán 2016-2019. Recuperado en http://www.sutamarchan-boyaca.gov.co/index.shtml?apc=gbxx--2654087\&sh_itm=fb2bbf 07efb6388631b1d499a716c03a\&sel_ids=1

Alcaldía de Tibaná. (2016). Plan de desarrollo Tibaná 2016-2019. Recuperado de http://tibanaboyaca.gov.co/apc-aa-files/33343662623634323734356430633336/plan-de-desarro1lo-2016-2019-1-.pdf

Alcaldía de Tota. (2016). Plan de desarrollo de Tota 2016-2019. Recuperado de http://www.totaboyaca.gov.co/index.shtml\#2

Alcaldía Mayor de Bogotá. (2016). Cundinamarca, Boyacá, Meta y Tolima, la despensa de Bogotá. Recuperado de http://www.bogota.gov.co/article/temas-de-ciudad/desarrollo-economico/ cundinamarca-boyaca-meta-y-tolima-la-despensa-de-bogota

Alcaldía Mayor de Tunja (2016). Plan de desarrollo Tunja 2016-2019. Recuperado de http://tunjaboyaca.gov.co/apc-aa-files/39353939373461333461303765613162/pdm-tunja-en-equipo-2016-2019.pdf

Alcaldía Municipal de Aquitania. (2016). Plan de desarrollo de Aquitania 2016-2019. Recuperado de http://www.aquitania-boyaca.gov.co/index.shtml\#2

Alcaldía Municipal de Duitama (2016). Plan de desarrollo Duitama 2016-2019. Recuperado de http:// www.semduitama.gov.co/proyectos/PLAN\%20DE\%20DESARROLLO\%20ACUERDO\%20013.pdf

Alcaldía Municipal de Jenesano. (2016). Plan de desarrollo Jenesano 2016-2019. Recuperado de http://www.jenesano-boyaca.gov.co/index.shtml

Alcaldía Municipal de Nobsa. (2016). Plan de desarrollo Nobsa. Territorio sin límites, 2016-2019. Recuperado de http://www.nobsa-boyaca.gov.co/index.shtml?apc=I-xx-1-\&x=1923296

Alcaldía Municipal de Nuevo Colón. (2016). Plan de desarrollo de Nuevo Colón 2016-2019. Recuperado de http://www.nuevocolon-boyaca.gov.co/index.shtml? $\mathrm{x}=2801646$

Alcaldía Municipal de Paipa (2016). Plan de desarrollo Paipa construcción colectiva bienestar para todos. Recuperado de http://www.concejo-nobsa-boyaca.gov.co/es/novedades/boletines-ypublicaciones/661-plan-de-desarrollo-2016-2019 
Alcaldía Municipal de Ráquira. (2016). Plan de desarrollo municipal 2016-2019. Porque Ráquira merece seguir creciendo. Recuperado de http://www.raquira-boyaca.gov.co/index.shtml?x=2737489

Alcaldía Municipal de Sáchica. (2016). Plan de desarrollo municipal de Sáchica 2016-2019. Recuperado de http://www.sachica-boyaca.gov.co/index.shtml?apc=I-xx--1871059\&x=1869385

Alcaldía Municipal de Tibasosa. (2016). Plan de desarrollo municipal de Tibasosa 2016-2019. Recuperado de http://www.tibasosa-boyaca.gov.co/index.shtml\#5

Alcaldía Municipal de Villa de Leyva (2016). Plan de desarrollo municipal Villa de Leyva. Recuperado de http://www.villadeleyva-boyaca.gov.co/MiMunicipio/Programagobierno/Plan\%20 de\%20Desarrollo\%20Municipal\%202016\%20-\%202019.pdf

Cárdenas, C. (2015). Exportación de productos no tradicionales del departamento de Boyacá (ensayo de grado). Relaciones Internacionales, Seguridad y Estrategia, Universidad Militar, Bogotá, Colombia.

Centro de Aprendizaje de Finanzas e Inversiones Rurales y Comité Nacional de Impulso Colombia. (2014). Definición de la agricultura familiar para Colombia. Colombia: Comité Nacional de Impulso Colombia.

Corporación Autónoma Regional de Boyacá. (2015). Fortalecimiento y promoción de sistemas de producción limpia en el sector agroindustrial del bocadillo en el municipio de Moniquirá-Boyacá. Tunja, Colombia: Corpoboyacá.

De León, L., de León, E., Catalano, J., Rodríguez, D., Neira E., y Cuevas, R. (2004). Transporte rural de productos alimenticios en América Latina y el Caribe. Roma: Organización de las $\mathrm{Na}-$ ciones Unidas para la Agricultura y la Alimentación.

Departamento de Planeación Nacional. (2013). Caracterización social, territorial y de producción del DDRS y DNP del anuario estadístico del sector agropecuario (documento inédito). Bogotá: DPN.

Departamento Nacional de Planeación. (s. f.). Planes indicativos de acción reportados al DNP 20162019 (documento inédito). Bogotá: DNP.

Gobernación de Boyacá. (2016). Plan de desarrollo 2016-2019 Creemos en Boyacá, Tierra de Paz y Libertad. Recuperado de http://www.boyaca.gov.co/gobernacion/politicas-planes-y-programas/9498pdd-boyaca-2016-2019

Granados, A., y Guzmán, A. (2003). Plan estratégico de recolección, empaque y distribución de papa y cebolla en el tramo de Boyacá-Bogotá (tesis de grado). Facultad de Ingeniería, Pontificia Universidad Javeriana, Bogotá, Colombia.

Lozano, I., y Restrepo, J. (2015). El papel de la infraestructura rural en el desarrollo agrícola en Colombia. Borradores de Economía, 904, 2-38.

MinAgricultura y Banco Agrario crean Agrolisto para fomentar el acceso al crédito. (2017). Agronet. Recuperado de http://www.agronet.gov.co/Noticias/Paginas/MinAgricultura-y-Banco-Agrario-crean-Agrolisto-para-fomentar-el-acceso-al-credito---.aspx

Ministerio de Agricultura e ICA. (1995). Censo de minifundio en Colombia. Bogotá. Ministerio de Agricultura.

Ministerio de Agricultura. (2016). Colombia siembra. El plan en el que el campo es protagonista. Recuperado de http://www.cenfer.co/agroferia/media/COLOMBIA_SIEMBRA_2016.pdf 
Ministerio de Agricultura. (s. f.). Lineas de financiamiento. Recuperado de http://www.agronet.gov. $\mathrm{co} /$ capacitacion/Paginas/PequenosProductores/credito-agropecuario.aspx

Ministerio de Agricultura. (s. f.). Programa desarrollo rural con equidad-DRE. Recuperado de https://www.minagricultura.gov.co/ministerio/programas-y-proyectos/Paginas/ProgramaDesarrollo-Rural-con-Equidad-DRE.aspx

Ministerio de Transporte. (2014). Costo por movilización y por tiempos logísticos (ajuste ACPM definitivo). Recuperado de https://www.mintransporte.gov.co/Documentos/documentos_del_ ministerio/regulacion_economica

Resolución 184 de 2009. Por la cual se establecen las tarifas correspondientes a los operadores y usuarios de transporte ferroviario de carga y pasajeros en el Corredor del Sistema Ferroviario Central. Diario Oficial n. ${ }^{\circ}$ 47.234, 16 de enero de 2009.

World Economic Forum y McKinsey \& Company (2010). Realizing a new vision for agriculture: $a$ roadmap for stakeholders. Suiza: WEF. 